\title{
Sepsis and neonatal hypoxic ischemic encephalopathy
}

\begin{abstract}
Introduction: hypoxic-ischemic encephalopathy is a clinical syndrome due to lack of perfusion and oxygen to the brain. One of the main risk factors for its development is perinatal infection, which also increases the risk of sepsis and worsens the patient's prognosis.

Objective: to determine the relationship between sepsis and hypoxic-ischemic encephalopathy in patients undergoing regional brain hypothermic therapy.

Methodology: retrospective, descriptive study of a cohort of newborns with hypoxicischemic encephalopathy subjected to brain hypothermic therapy for whom clinical, neurological and paraclinical variables for the study of sepsis were compared, and for which the probability of sepsis was estimated using the Phillips scale.

Results: $85.7 \%$ of the patients presented some sentinel event, the most frequent being prolonged expulsion (55.6\%). 90\% of the patients presented with moderate and severe Sarnat-Sarnat classification. $65 \%$ of the patients received antibiotics and two patients had positive blood cultures. $76.1 \%$ of the patients with blood cultures had negative results. Only two patients (3.1\%) had a score of two on the Phillips scale. A relationship was found with respect to the outcome, with a p value of 0.16 , and 0.175 between the Phillips score and the severity of the encephalopathy. The relationship between clinical severity according to the Sarnat- Sarnat scale and positive blood culture had a p value of 0.051 .

Conclusion: it is very difficult to determine the presence of infection with any degree of certainty in these patients because their pathological process behaves very similarly to sepsis and this, added to inflammatory mediators and physiological responses, make it difficult to predict the risk of sepsis using current scales. It is necessary to find markers that are more sensitive and specific for sepsis in neonates undergoing hypothermia in order to improve the decision-making process and its effects on the prognosis for these patients.
\end{abstract}

Keywords: Hypoxia-Ischemia, Brain, Hypothermia, Sepsis
Volume 4 Issue 2 - 2016

\author{
Monica D 'Amato Gutierrez,' Andrés Felipe \\ Uribe, ${ }^{2}$ Carolina Serrano Tabares ${ }^{3}$ \\ 'Department of Pediatrics, Universidad Pontificia Bolivariana, \\ Colombia \\ ${ }^{2}$ Neonatal Intensive Care Unit, Clinica Universitaria Bolivariana, \\ Colombia \\ ${ }^{3}$ Department of Pediatrics, Universidad, Clinica Universitaria \\ Bolivariana, Colombia
}

Correspondence: Carolina Serrano Tabares, Street 78b No. 72a - 109, Medellin, Antioquia, Colombia, Tel 4936300 - ext. 9842 \& 3006203735, Email caropalsa@gmail.com

Received: July 18, 2015 | Published: January 29, 2016
Abbreviations: HIE, Hypoxic-Ischemic Encephalopathy; OTI, Orotracheal Intubation; CRP, C-Reactive Protein; PMN, Polymorphonuclear (Neutrophils); BC, Blood Cultures; PCR, Polymerase Chain Reaction; GE, Gestational age; CP, Cephalic Perimeter

\section{Introduction}

One of the main pathologies that have a major impact on the morbidity and mortality of pediatric patients is hypoxic-ischemic encephalopathy (HIE), a clinical syndrome caused by a lack of perfusion and oxygen to the brain, that manifests with clinical, paraclinical and radiologic signs in the brain and in other organs. Global incidence is described as 1-3 per every 1000 full-term or near full term (greater than 36 weeks) neonates. To date, the only therapy that has shown a decrease in neuronal damage and makes it possible to apply neuroprotection tools is hypothermic therapy. In Colombia, there are no reports about the incidence of this pathology but it is known that it has been increasing, as has the interest in finding therapeutic measures to reduce immediate complications and neurological sequelae. ${ }^{1-6}$

There are several risk factors that might lead to the development of HIE and one of the most important is perinatal infection. It has been shown to increase the risk of developing HIE due to the inflammatory response that is produced, and causes brain damage. ${ }^{7-13}$ In addition to being a factor for HIE, infection in the neonate can cause sepsis and that can worsen the patient's prognosis. At Clinica Bolivariana de Medellin, selective brain hypothermic therapy has been implemented since 2011, and this study was designed in order to evaluate the presence of sepsis in these patients and its relationship to clinical severity.

\section{Materials and methods}

\section{General objectives}

to determine the relationship between sepsis and hypoxic-ischemic encephalopathy in patients undergoing regional brain hypothermic therapy.

\section{Specific objectives}

- Describe the general characteristics of the population

- Describe the average of leukocytes, absolute value of neutrophils, and CRP

- Describe the relationship within the severity of the HIE measured according to the modified Sarnat-Sarnat scale and the presence of positive $\mathrm{BC}$ upon admission.

- Describe the relationship between the severity of the HIE measured according to the modified Sarnat- Sarnat scale and the stratification scores using paraclinical criteria (Phillips score) for sepsis. 
- Describe the relationship between the $\mathrm{BC}$ results, the total Phillips score, and the patient's outcome.

A retrospective, descriptive cohort study was designed.

After obtaining authorization from the institution, we collected data from the clinical histories of all patients diagnosed with HIE that were subjected to selective brain hypothermic therapy between January 2011 through April 2015 at Clinica Universitaria Bolivariana, in Medellin. No patients were excluded.

Data for 63 patients were gathered. Variables for basal characteristics such as gestational age, weight at birth, type of delivery, sex, sentinel event, Apgar scale at 1 and 5 minutes, and Sarnat- Sarnat classification were analyzed in addition to clinical variables such as the presence of seizures, and presence or absence of an epileptic status, the need for intubation during the first hour of life, time when effective respiration and suction were acquired, and patient's outcome such as discharged, referred or deceased. Paraclinical variables such as leukocytes, neutrophils, lymphocytes, bands, CRP, and BC were also evaluated. The data for the paraclinical tests were taken during the first 10 hours of life, as part of the protocol for hypothermia.

The Phillips scale, which estimates the probability of sepsis in the total score is greater than two, was selected (Table 1).

Table I Phillips Scale

\begin{tabular}{ll}
\hline Test & Score \\
\hline PMN $<1750 / \mathrm{mm}$ & $\mathrm{I}$ \\
Leukocytes $<5000$ or $>40000$ & $\mathrm{I}$ \\
Band ratio/ PMN $>0.2$ & $\mathrm{I}$ \\
$>0,4$ & 2 \\
CRP $>$ Img/dl & $\mathrm{I}$ \\
$>5 \mathrm{mg} / \mathrm{dl}$ & 2 \\
Screening for late sepsis is considered positive if $>2$ points
\end{tabular}

*PMN: Polymorphonuclear (Neutrophils); CRP: C-Reactive Protein. ${ }^{13}$

The frequencies granted for central trend and dispersion are described, according to whether they were measured as quantitative or qualitative variables, respectively. The univariate analysis was carried out using sepsis measured using the total Phillips score as the main outcome variable. Chi-square was used for the bivariate analysis of the dichotomous variables. All analyses were performed using a 0.05 level of significance $(\mathrm{p}<0.05)$. The statistical analysis program SPSS version 18 was used for tabulation and statistical analysis.

\section{Results}

The majority of patients were male $(69.8 \%)$ and full-term neonates, with an average weight at birth of $3037 \mathrm{~g}$ (Table 2A).

Table 2A General characteristics. Weight, gestational age, and cephalic perimeter

\begin{tabular}{llllll}
\hline & N & \multicolumn{4}{l}{ Minimum } \\
\hline GE (weeks) & 63 & 34.5 & 41 & $36+6$ & 0,49 \\
Weight (gr) & 63 & 2130 & 4280 & 3037 & 429,8 \\
CP (cm) & 27 & 31,5 & 37,0 & 33,9 & 1,3 \\
\hline
\end{tabular}

GE: gestational age. CP: cephalic perimeter.

All patients had institutional delivery, mostly in the second and third level complexity clinics in Medellin, and $82.5 \%$ of patients were born outside of our institution. Over $90 \%$ of the patients had moderate and severe Sarnat -Sarnat classifications, $47.6 \%$ and $46 \%$ respectively. Of the total number of patients, $54(85.7 \%)$ had some sentinel event, but less than $10 \%$ had more than one sentinel event. The most frequent sentinel event was the presence of prolonged expulsion in $55.6 \%$ of the patients, followed by meconium grade II or III in $20.6 \%$, coiling of the cord in $14.3 \%$, hemorrhage as abruptio or placenta previa in $9.5 \%$, and uterine rupture $6.3 \%$. (Table $2 \mathrm{~B}$ )

Table 2B General characteristics. Type of delivery, sentinel event,Apgar scale at I and 5 min., Sarnat- Sarnat classification

\begin{tabular}{|c|c|c|}
\hline Characteristic & Frequency & Percentage (\%)* \\
\hline \multicolumn{3}{|l|}{ Place of birth } \\
\hline I. Our Clínic & II & 17,5 \\
\hline 2. Other institution-Medellin & 30 & 47,6 \\
\hline 3. Institution outside of Medellin & 17 & 27 \\
\hline 4. Non-institutional delivery & 0 & 0 \\
\hline No data & 5 & 7,9 \\
\hline \multicolumn{3}{|l|}{ Sex } \\
\hline I. Female & 18 & 28,6 \\
\hline 2. Male & 44 & 69,8 \\
\hline \multicolumn{3}{|l|}{ Delivery } \\
\hline I.Vaginal spontaneous & 37 & 58,7 \\
\hline 2.Vaginal induced & 1 & 1,6 \\
\hline 3. Instrument assisted & 8 & 12,7 \\
\hline 4. Cesarean section & 17 & 27 \\
\hline \multicolumn{3}{|l|}{ Sentinel event } \\
\hline I. Meconium & 13 & 20,6 \\
\hline 2. Hemorrhage & 6 & 9,5 \\
\hline 3. Coiling of the cord & 9 & 14,3 \\
\hline 4. Prolonged expulsion & 35 & 55,6 \\
\hline 5. Uterine rupture & 4 & 6,3 \\
\hline \multicolumn{3}{|l|}{ Apgar scale } \\
\hline \multicolumn{3}{|l|}{ I minute/ 5 minutes } \\
\hline 0 & $12 / 5$ & $19 / 7,9$ \\
\hline I & $12 / 4$ & $19 / 6,3$ \\
\hline 2 & $20 / 14$ & $31 / 22$ \\
\hline 3 & $10 / 4$ & $15,9 / 6,3$ \\
\hline 4 & $5 / 14$ & $7,9 / 22$ \\
\hline 5 & $\mathrm{I} / \mathrm{I}$ & $1,6 / 17,5$ \\
\hline 6 & $0 / 3$ & $0 / 4,8$ \\
\hline 7 & $0 / 2$ & $0 / 3,2$ \\
\hline 8 & $0 / 0$ & $0 / 0$ \\
\hline 9 & $0 / 0$ & $0 / 0$ \\
\hline 10 & $0 / 2$ & $0 / 3,2$ \\
\hline No data & $3 / 4$ & $4,8 / 6,3$ \\
\hline \multicolumn{3}{|l|}{ Sarnat and Sarnat classification } \\
\hline Mild & 3 & 4,8 \\
\hline Moderate & 30 & 47,6 \\
\hline Severe & 30 & 47,6 \\
\hline
\end{tabular}

*Percent of total patients.

The 1 minute Apgar scale for all patients had a score of five or less and, although at I5 minutes there were patients with scores above seven, most patients ( $82 \%$ ) had scores of five or less (Table 2B).

Most patients had to be intubated at birth (88.9\%). 66.7\% of the patients were able to maintain spontaneous respiration in the first two weeks of life (Table 3).

Table 3 Clinical variables

\begin{tabular}{lll}
\hline Characteristic & Frequency & Percentage (\%)* \\
\hline First clinical seizure & & \\
\hline I. $<6$ hours & 18 & 28,6 \\
2. 6-48 hours & 27 & 42,9 \\
3. $>48$ hours & 3 & 4,8 \\
\hline
\end{tabular}


Table Continued...

\begin{tabular}{lll}
\hline Characteristic & Frequency & Percentage (\%) \\
\hline First clinical seizure & & \\
\hline 4. Did not seize & 13 & 20,6 \\
Seizure status & 7 & $1 \mathrm{I}, \mathrm{I}$ \\
Seizure crisis after hypothermia & 7 & $1 \mathrm{I}, \mathrm{I}$ \\
OTI I st houra & 56 & 88,9 \\
Respirationb & & \\
I. Immediately at birth & 4 & 6,3 \\
2. <2 weeks & 42 & 66,7 \\
3. 2- 4 weeks & 7 & $1 \mathrm{I}, \mathrm{I}$ \\
4. >4 weeks & 4 & 6,3 \\
5. Not achieved & 3 & 4,8 \\
Suctionc & & \\
I. <2 weeks & 18 & 28,6 \\
2. 2 a 4 weeks & 26 & 41,3 \\
3. >4 weeks & 7 & $1 \mathrm{I}, \mathrm{I}$ \\
4. Not achieved-gastrostomy & 8 & 12,7 \\
Final outcome & & 87,3 \\
I. Discharged-alive & 55 & 6,3 \\
2. Referral-alive & 4 & 6,3 \\
3. Deceased & 4 & \\
& &
\end{tabular}

*Percent of total patients.

aOTI: orotracheal intubation during the first hour of life.

btime of acquisition of successful spontaneous respiration.

ctime of acquisition of effective suction for oral feeding.

When evaluating the moment when adequate suction for safe and effective feeding was achieved, $41.3 \%$ of patients achieved it between the second and fourth weeks of life $(12.7 \%$ of patients did not have complete criteria for safe feeding, including patients that required gastrostomy and the patients who died) (Table 3).

$82.5 \%$ of the patients were discharged to home, $4(6.3 \%)$ were referred to other institutions due to the need for other specialties, and 4 patients $(6.3 \%)$ died (Table 3$)$.

Laboratory values are specified in tables 4, 5 and 6. Leukocyte counts were as low as $6829 / \mathrm{ml}$ and as high as $38210 / \mathrm{ml}$; no patient had neutropenia and only five patients had platelets below 100000/mm3 $(3.15 \%)$ during the first 6 hours. Most patients did not have bands (95\%), giving a band/neutrophil average ratio of 0.014 . Average CRP was $0.8 \mathrm{mg} / \mathrm{dl}$, and only 2 patients $(3.1 \%)$ had results over $5 \mathrm{mg} / \mathrm{dl}$ (9.31 y $6.1 \mathrm{mg} / \mathrm{dl})$.

Of the 51 patients for whom $\mathrm{BC}$ were obtained (80.9\%), 76.1\% were negative.

Of the total number of patients evaluated, 41(65\%) received antibiotics before or during hypothermic therapy and only two (3.17\%) had positive BC for Streptococcus agalactiae. The other patient whose BC was positive for Escherichia coli did not receive antibiotics before or during hypothermic therapy.

On the Phillips scale, only two (3.1\%) patients had a score of 2 , both were alive at the end of their hospitalization, and both received antibiotics before or during hypothermic therapy. One of the deceased patients had a Phillips score of 1 and the other 2 had scores of zero. This relationship had a $p$ value of 0.16 between the Phillips score and the final outcome, and a $p$ value of 0.175 for the relationship between the Phillips score and the severity of the HIE (Table 7 and 8, and Graph 1).

Using Pearson's chi-square test, a p value of 0.051 was found [for the relationship] between the Sarnat - Sarnat moderate and severe classification, and positive BC (Table $9 \&$ Graph 1).
Table 4 Hemoleucogram and C reactive protein (CRP)

\begin{tabular}{llllll}
\hline & N & Minimum & Maximum & Average & $\begin{array}{l}\text { Standard } \\
\text { deviation }\end{array}$ \\
\hline Hemoglobin & 45 & 8.5 & 20.9 & 15.1 & 2.85 \\
Hematocrit & 46 & 23 & 65 & 45.69 & 8.8 \\
Leukocytes/ml & 61 & 6829 & 38210 & $206 \mid 4.38$ & 7908 \\
\% PMN & $6 I$ & 26.5 & 92 & 64.5 & 14.27 \\
PMN.Absolute/ml & 63 & 2433 & 25176 & 13257 & 5.97 \\
\% Lymphocytes & 61 & 1.2 & 69 & 26,3 & 16.084 \\
Platelets/mm3 & 61 & 90000 & 196800 & 179000 & 31686.8 \\
Bands\% & 59 & 0 & 20 & 0.85 & 3.05 \\
Bands abs & 63 & 0,0 & 5311 & 207.64 & 788.34 \\
Bands/PMN & 63 & 0,0 & 0,31 & 0.014 & 0.05 \\
CRP & 60 & 0 & 9.31 & 0.803 & 1.46 \\
\hline
\end{tabular}

*PMN: polymorphonuclear (neutrophils); CRP: C-reactive protein.

Table 5 Blood Cultures

\begin{tabular}{lll}
\hline $\begin{array}{l}\text { Characteristic } \\
\text { Blood Culture }\end{array}$ & \multicolumn{2}{l}{ Frequency } \\
\hline Positive & 3 & 4,8 \\
Multi-sensitive E. coli & 1 & 1,6 \\
Penicillin S S. agalactiae & 2 & 3,2 \\
Negative & 48 & 76,1 \\
Not performed & 12 & 19 \\
\hline
\end{tabular}

Table 6 Phillips score

\begin{tabular}{lll}
\hline Characteristic & Frequency & Percentage (\%) \\
\hline PMN $<$ I750/ml & 0 & 0 \\
Leucocytes /ml & & \\
$<5000$ & 0 & 0 \\
$>40000$ & 0 & 0 \\
Band/PMN & & \\
$<0.2$ & 61 & 95.2 \\
$>0.2$ & 2 & 3,2 \\
$>0.4$ & 0 & 0 \\
CRP (mg/dl) & & \\
$<$ I & 43 & 68.3 \\
$>\quad$ I & 16 & 23.8 \\
$>\quad 5$ & 2 & 3.2 \\
No data & 3 & 4,8 \\
Total score & & \\
0 & 44 & 69,8 \\
I & 17 & 27 \\
2 & 2 & 3,2 \\
\hline
\end{tabular}

PMN: Polymorphonuclear (neutrophils); CRP: C Reactive Protein.

Table 7 Relationship between total Phillps score and outcome

\begin{tabular}{|c|c|c|c|c|c|c|c|}
\hline & & & \multicolumn{4}{|c|}{ Outcome } & \multirow[b]{2}{*}{ Total } \\
\hline & & & No data & $\begin{array}{l}\text { Alive- } \\
\text { Discharged }\end{array}$ & $\begin{array}{l}\text { Alive- } \\
\text { Referred }\end{array}$ & Deceased & \\
\hline \multirow{2}{*}{\multicolumn{2}{|c|}{0}} & \multirow{2}{*}{$\begin{array}{l}\text { Number } \\
\%\end{array}$} & 3 & 35 & 3 & 3 & 44 \\
\hline & & & $100 \%$ & $67,3 \%$ & $75,0 \%$ & $75,0 \%$ & $69,8 \%$ \\
\hline \multirow{4}{*}{$\begin{array}{l}\text { Total } \\
\text { Phillips }\end{array}$} & \multirow{2}{*}{1} & Number & 0 & 16 & 0 & 1 & 17 \\
\hline & & $\%$ & $0 \%$ & $30,8 \%$ & $0 \%$ & $25 \%$ & $27 \%$ \\
\hline & \multirow{2}{*}{2} & Number & 0 & 1 & 1 & 0 & 2 \\
\hline & & $\%$ & $0 \%$ & $1,9 \%$ & $25,0 \%$ & $0 \%$ & $3,2 \%$ \\
\hline \multirow{2}{*}{ Total } & & Number & 3 & 52 & 4 & 4 & 63 \\
\hline & & $\%$ & $100 \%$ & $100 \%$ & $100 \%$ & $100 \%$ & $100 \%$ \\
\hline
\end{tabular}


Table 8 Relationship between HIE and total Phillips score

\begin{tabular}{lllll}
\hline & \multicolumn{4}{c}{ Total Phillips } \\
& 0 & I & 2 & Total \\
\hline Mild & I & 2 & 0 & 3 \\
Moderate & 24 & 4 & 2 & 30 \\
Severe & 19 & II & 0 & 30 \\
Total & 44 & 17 & 2 & 63 \\
\hline
\end{tabular}

Table 9 Relationship between HIE severity measured by the Sarnat-Sarnat scale and the presence of positive blood culture upon admittance

\begin{tabular}{llllll}
\hline \multirow{2}{*}{ Sarnat-Sarnat Scale } & \multicolumn{2}{l}{ Blood Culture } & \multicolumn{2}{c}{ Total } \\
& Negative & Positive & Not performed & \\
\hline \multirow{4}{*}{ Mild } & I & 0 & 2 & 3 \\
& Moderate & 22 & 0 & 8 & 30 \\
& Severe & 23 & 3 & 4 & 30 \\
Total & 46 & 3 & 14 & 63 \\
\hline
\end{tabular}

\section{Discussion}

Most of our patients were males with an adequate weight for their gestational age, similar to what has been reported in different studies. As part of the institution's protocol for starting a patient on hypothermic therapy, the modified Sarnat and Sarnat classification must be moderate or severe. This explains why most patients had this classification, which correlates with what has been found by other authors. $^{\text {? }}$

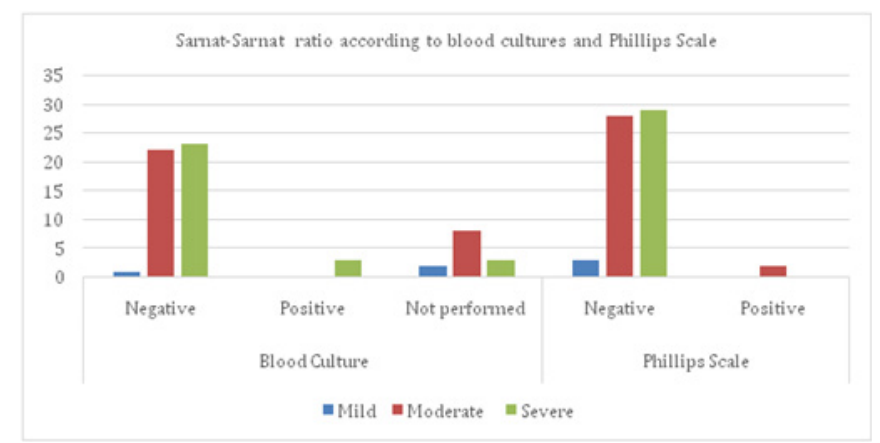

Graph I Sarnat-Sarnat ratio according to blood cultures and Phillips scale.

Infection associated with perinatal hypoxia is not only a risk factor, but also becomes a causal factor in some cases and a factor for a poor prognosis for increased brain damage. Chorioamnionitis is the main maternal infection and occurs in up to $32 \%$ of full term and preterm births. The most common germs described include group B Streptococcus, with a rate of infection of $50 \%$ of babies born to mothers colonized by this germ $(15-25 \%)$. These patients are described as having a lower Apgar score at birth, and up to $37 \%$ develop childhood cerebral palsy. ${ }^{1,9-11}$

Most of our patients had low Apgar scores, which is similar to the findings by authors such as Hermansen \& Hermansen, ${ }^{11}$ with $20 \%$ of children born to mothers with uterine infections having a low Apgar at 1 minute of life, and $3 \%$ at 5 minutes of life.

In our study, after a thorough review of the clinical histories, we were not able to identify for most patients whether the mother was the cause of infection. Most of our patients had prolonged expulsion either as a single sentinel event or associated with others. This leads to more contact between the baby and the mother's blood and mucus in the birth canal and thus increases the probability of transmitting an infection, if there is one.
Some authors have attempted to establish laboratory value scales to increase the probability of diagnosing sepsis in the patients, especially when $\mathrm{BC}$ results are negative or not available. ${ }^{11}$ Newman et al. ${ }^{14}$ did a study in which they evaluated several ranges of laboratory values and their likelihood ratio for the probable sepsis diagnosis. However, that scale has not been validated. Some others have described the use of scales such as Phillips', which was used in this study. ${ }^{7,11,15,16}$

No patient presented leukocytosis or leukopenia according to the Phillips score. Most patients showed leukocytosis as a total value greater than $25.000 / \mathrm{ml}$. With respect to the presence of bands, only $6.2 \%$ of the patients had bands during their first hemoleucogram. The CRP was also low in most patients, and patients with higher values defined the score of 2 on the Phillips scale and it was used as a paraclinical criterion for sepsis. Our results for CRP are comparable to the results obtained by Tann et al. ${ }^{7}$ where they found that in patients with confirmed bacteremia there was a $22 \%$ elevation and CRP from the first day of life, compared to the control group not diagnosed with sepsis in which the elevation was only present in $5 \%$ of patients. The study by Okumus et al. ${ }^{17}$ is also similar. In that study, they found that patients with HIE on hypothermic therapy had a higher blood CRP when compared to the controls.

The limitations for $\mathrm{BC}$ in neonates include the fact that is not always possible to obtain an adequate sample volume, there might not be enough bacterial load circulating in the blood, the use of antibiotics by the mother before childbirth which can alter the positivity, and some slow-growing microorganisms. All of this makes it difficult to diagnose sepsis in neonates. ${ }^{7,11,14}$

In our study, $4.8 \%$ of the patients had positive $\mathrm{BC}$, similar to Tann et al. $^{7}$ with a $3.5 \%$ prevalence of bacteremia, and $8.9 \%$ when findings such as PCR were added. The germs found were similar to the most common ones reported in local and international literature, with Streptococcus agalactiae being the most frequent., 71,14,18,19

One way to improve the detection of bacteremia is to perform PCR. However, this is not routinely done in our institution because it is not included in the protocol for sepsis. Whether it can be justified in circumstances other than research, would be the subject for another study because, in light of the multiple noxa found in asphyctic patients, and to the changes in the paraclinical tests as a result of hypothermia, it might be justified in the future for this group of patients. ${ }^{7}$

The limitations found in the study were that the data gathered from the clinical histories of patients not born in our institution were incomplete and the background information was insufficient.

\section{Conclusion}

The correlation between perinatal infection and HIE has been described at length, but the true prevalence of coexistence of the two processes varies according to the different studies. This proves once again that it is very difficult to determine the presence of infection with any degree of certainty, especially patients on hypothermic therapy that are coming from a pathological process that behaves very similarly to this disease. Some specific circumstances of these patients, such as the presence of inflammatory mediators, counterregulatory physiological responses, changes to the metabolic variables due to the hypothermia, and modified cell migration, make it necessary to reevaluate the scales that are currently used to predict the risk of sepsis and, therefore, the start of empirical antibiotics, in light of a special group of patients. All of the above is reflected by the large variability of the results found in the literature in studies like ours. Therefore, it is extremely important to find markers with greater sensitivity and specificity for sepsis in 
neonates subjected to hypothermia, to improve decision-making and the impact on the prognosis for these patients. The extensive use of antibiotics in these patients (65\% of the total) could be decreased by using more specific tools to diagnose sepsis.

\section{Acknowledgements}

Luz Aida Mejia C. Nurse. MSC in Epidemiology. Medellin, Colombia.

\section{Conflicts of Interest}

We assert that no conflicts of interest exist.

\section{References}

1. Wu YW. Systematic review of chorioamnionitis and cerebral palsy. Ment Retard Dev Disabil Res Rev. 2002;8(1):25-29.

2. Lai MC, Yang SN. Perinatal hypoxic-ischemic encephalopathy. $J$ Biomed Biotechnol. 2011;2011:609813.

3. Schulzke SM, Rao S, Patole SK. A systematic review of cooling for neuroprotection in neonates with hypoxic-ischemic encephalopathy-are we there yet? BMC Pediatr. 2007;5(7):30.

4. Shankaran S, Laptook AR, Ehrenkranzetal RA, et al. Whole body hypothermia for neonates with hypoxic-ischemic encephalopathy. $N$ Engl J Med. 2005;353(15):1574-1584.

5. Edwards AD, Azzopardi DV. Therapeutic hypothermia following perinatal asphyxia. Arch Dis Child Fetal NeonatalEd.2006;91(12):F127F131

6. Gancia P, Pomero G. Therapeutic hipotermia in the prevention of hypoxic- ischaemic encephalopathy: new categories to be enrolled. $J$ Matern Fetal Neonatal Med. 2012;25(Suppl 4):94-96.

7. Tann CJ, Nkurunziza P, Nakakeeto M, et al. Prevalence of Bloodstream Pathogens Is Higher in Neonatal Encephalopathy Cases vs. Controls Using a Novel Panel of Real-Time PCR Assays. PLoS ONE. 2014;9(5):e97259.
8. Eklind S, Mallard C, Leverin AL, et al. Bacterial endotoxin sensitizes the immature brain to hypoxic-ischaemic injury. Eur $J$ Neurosci. 2001;13(6):1101-1106.

9. Stridh L, Mottahedin A, Johansson ME, et al. Toll-Like Receptor-3 Activation Increases the Vulnerability of the Neonatal Brain to HypoxiaIschemia. J Neurosci. 2013;33(29):12041-12051.

10. Zhao J, Chen Y, Pi G. Effect of intrauterine infection on brain development and injury. Int J Devl Neuroscience. 2013;31(7):543-549.

11. Hermansen MC, Hermansen MG. Perinatal infections and cerebral palsy. Clin Perinatol. 2006;33(2):315-333.

12. Girard S, Kadhim H, Roy M, et al. Role of perinatal inflammation in cerebral palsy. Pediatr Neurol. 2009;40(3):168-174.

13. Phillip AG, Hewitt JR. Early diagnosis of neonatal sepsis. Pediatrics. 1980;65(5):1036-1040.

14. Newman TB, Puopolo KM, Wi S, et al. Interpreting complete blood counts soon after birth in newborns at risk for sepsis. Pedaitrics. 2010;126(5):903-909.

15. http://gpc.minsalud.gov.co/Documents/Guias-PDF-Recursos/RN\%20 sano/GPC_Prof_Sal_RNSano.pdf

16. Christensen RD, Henry E, Jopling J, et al. The CBC: reference ranges for neonates. Semin Perinatol. 2009;33(1):3-11.

17. Okumus N, Beken S, Aydin B, et al. Effect of therapeutic hypothermia on $\mathrm{C}$-Reactive protein levels in patients with perinatal asphyxia. $\mathrm{Am} \mathrm{J}$ Perinatol. 2015;32(7):667-674.

18. Camacho-Gonzalez A, Spearman PW, Stoll BJ. Neonatal infectious diseases: evaluation of neonatal sepsis. Pediatr Clin North Am. 2013;60(2):367-89.

19. Wynn JL, Wong HR. Pathophysiology and treatment of septic shock in neonates. Clin Perinatol. 2010;37(2):439-479. 